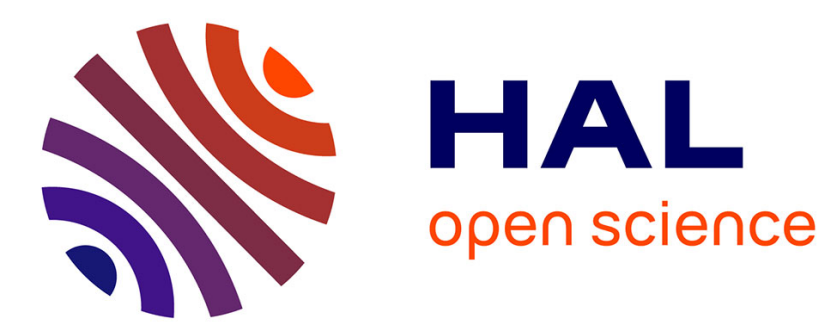

\title{
Improvement of X-ray castings inspection reliability by using Dempster-Shafer data fusion theory
}

\author{
Ahmad Osman, Valerie Kaftandjian, Ulf Hassler
}

\section{To cite this version:}

Ahmad Osman, Valerie Kaftandjian, Ulf Hassler. Improvement of X-ray castings inspection reliability by using Dempster-Shafer data fusion theory. Pattern Recognition Letters, 2011, 32, pp.168-180. hal-00878528

\section{HAL Id: hal-00878528 \\ https://hal.science/hal-00878528}

Submitted on 13 Nov 2013

HAL is a multi-disciplinary open access archive for the deposit and dissemination of scientific research documents, whether they are published or not. The documents may come from teaching and research institutions in France or abroad, or from public or private research centers.
L'archive ouverte pluridisciplinaire HAL, est destinée au dépôt et à la diffusion de documents scientifiques de niveau recherche, publiés ou non, émanant des établissements d'enseignement et de recherche français ou étrangers, des laboratoires publics ou privés. 


\section{Improvement of $\mathbf{X}$-ray castings inspection reliability by using Dempster-Shafer data fusion

12Abstract:

13

14The aim of this work is to improve the classification of defects in X-ray inspection by developing a 15 new method based on Dempster-Shafer data fusion theory where measured features on the detected 16objects are considered as information sources. From the histogram of features values on a learning 17 database of manually classified objects, an automatic procedure is proposed to define a set of mass 18 functions for each feature. The spatial repartition of features is divided into regions of confidence 19with corresponding mass functions. A smooth transition between regions is ensured by using fuzzy 20membership functions. The whole process is carried out without any expert intervention. Validation 21 takes place on a testing database. Data fusion leads to a significant improvement of classification 22performances with respect to the actual system.

23

24Key words: X-ray imaging, castings inspection, true defects, false alarms, features extraction, data 25fusion, confidence levels, mass value, Dempster-Shafer theory.

\section{Introduction:}

29X-ray inspection is increasingly used as a tool for non destructive testing of industrial parts, such as 30aluminium castings in the automotive sector. In this field, new materials or processes are developed 31 for cost and weight optimization. As those materials are operated near their load limit, it is neces32sary to check each produced part thoroughly. One main difficulty in X-ray castings inspection is the 33 detection of false alarms (or false defects), especially if very small and low contrasted defects have 34 to be detected. Therefore, efforts have to be made to reduce the rejection rate of good parts without 35risking to miss true defects. Once the X-ray images are processed for defect detection, the problem 36 can be reduced to a classification problem, in order to discriminate the true defects (TD) from the 37 false defects (FD).

38

Corresponding author: Tel. +33 47243 8061. Email: valerie.kaftandjian@insa-lyon.fr (V. Kaftandjian). 
39Several approaches have been proposed for the classification of true defects and false defects, using 40X-ray inspection.

41Filtering techniques adapted to specific defect shapes such as bidirectional template matching tech42niques were used by (Newman and Jain, 1994) for finding gross casting defects. The method is at43tractive but limited to specific defect types and time consuming.

44

45Neural networks were used by (Lawson and Parker, 1994) for the automated detection of discon46tinuities in X-ray images of welds. A big advantage of this approach is to learn the neural net on a 47limited number of defect samples. In another work (Bonser and Lawson, 1998) a defect enhance48ment technique is introduced using a laws filter matched for round defects and Kirsch oriented filter 49matched for longitudinal defects was reported. The authors concluded that the neural net is more 50sensitive to fine defects but gives a high false alarm rate, whereas the filtering approach is more re51liable and faster but less sensitive to small defects. Additional investigations of neural networks are 52described in (Dobrazanski et al., 2005) for flaws in aluminium castings. The optimum network type 53was selected among multilayer perceptron (MLP), linear network, radial base functions network 54(RBF), generalized regression neural network (GRNN) and probabilistic neural network (PNN). 55The best results were obtained employing the PNN and MLP networks with performances of $90 \%$ 56 and $81 \%$ respectively on the test vectors, and $85 \%$ and $94.5 \%$ for the validation vectors. Perform57 ance is here given by the classification correctness of different defect types, but the false alarms rate 58 is not mentioned.

59

60In (Hernandez et al., 2004) the extracted features of each potential flaw were analysed and assigned 61 to one of the following classes: 'defect' or 'regular structure' by using a Neuro-Fuzzy method 62named ANFIS (Jang, 1993) which is used for constructing and training a fuzzy inference model that 63best classifies new data. The advantage of Neuro-Fuzzy systems is the combination of both proper64ties: non linear learning based on numerical data and handling uncertainties in data. However the 65 fuzzy model identification is very sensitive to which attributes are presented and class distribution 66in the training data. The developed system was used in casting and welds defects detection. The best 67 performance was obtained for aluminium castings with $95 \%$ of true defect classification (57 out of 6860 instances of defect class) and less than 1\% (199 non defects classified as defects out of 22876 69available) of false alarms rate. However the number of true defects on which the method is valid70 ated is small.

71

72Statistical pattern recognition was used in (Mery and Filbert, 2002) to classify castings defects. In 73statistical pattern recognition the classification is performed using the concept of similarity: similar 
74 patterns are assigned to the same class (Newman and Jain, 1994). Five classifiers were used by 75Mery: Linear classifier, Threshold classifier, Nearest neighbour classifier, Mahalanobis classifier 76and Bayes classifier. For each detected region, 71 features were extracted to characterise the poten77 tial flaws. The selection of the relevant features is made using sequential forward selection. Accord78ing to the authors, best performances were achieved with $100 \%$ of true defect classification and $7910 \%$ of false alarms rate for the Bayes classifier, and $94.7 \%$ of true defects classification and $4.1 \%$ 80 of false alarms respectively for the threshold classifier. However, the method is validated on only 8176 defects.

82

83A flexible inspection system called ISAR (Intelligent System for Automated Radioscopy) is presen84ted in (Fuchs, 2006). Defect classification is here performed by individual evaluation of character85 istic features of the segmented defect area, such as size, contrast, elongation. A special type of de86 fect classification was implemented by (Wenzel, 2003). Fourier coefficients are computed in order 87 to get translation- and rotation-invariant defect features. Those features can be assigned to a certain 88group, each representing a certain proper structure of the image. By computing the minimum dis89 tance of an actual defect region to each of the groups, artefacts generated at regular object structures 90may be recognized. Our study aims to improve the ISAR system performance, especially concern91ing the false alarm rate, by introducing data fusion of objects features.

92

93Data fusion theory was already applied by the authors in building a classification system, where the 94so-called confidence levels are assigned by a source to each detected object. The fusion between 95 confidence levels (or mass functions) is based on Dempster-Shafer theory (DS) and the final de96cision on the sample acceptability was done by a threshold in the confidence level. This approach 97was used in improving the detection of weld defects in (Kaftandjian et al, 2003), and in castings in98spection (Lecomte et al., 2006). The obtained results showed a more precise and reliable decision 99 by using the data fusion approach. However, the supervision of the expert was necessary to assign 100the confidence levels.

101

102In the present work, a new mass value attribution procedure is presented, which is completely auto103 matic, so that the expert supervision is no more necessary. Moreover, eleven features were com104 puted for each segmented structure in the casting (instead of four features in the previous study). 105Each feature is considered as a source of information. By using data fusion, the aim is to build an 106automatic classification system. To be able to fuse different sources, a common space where values 107 are comparable is necessary. An automatic method is introduced to convert from the space of fea108 ture values into the mass value space. 
110The rest of this paper is organized as follows. Section 2 introduces Dempster-Shafer data fusion 111 theory. Sections 3 and 4 present the proposed classification method and the performance measures. 112Afterwards experimental results are presented in section 5, discussed in section 6 before 113 concluding.

114

\section{Dempster-Shafer data fusion theory}

116

117Dempster-Shafer (DS) evidence theory was developed as an attempt to overcome the limitation of 118 conventional probability theory by handling uncertain, imprecise and incomplete information 119(Dempster, 1967; Shafer, 1976). It is also suited for combining information from different sources. 120In DS theory, a fixed set of $\mathrm{N}$ mutually exclusive and exhaustive elements, called the frame of 121 discernment, is defined that is symbolized by $\Theta=\left\{H_{1}, H_{2}, \ldots, H_{\mathrm{N}}\right\}$. The frame of discernment $\Theta$ 122defines the working space for the application being considered since it consists of all propositions 123 for which the information sources can provide evidence through using the so-called mass values. 124Information sources can also distribute mass values on subsets of the frame of discernment, $A_{i} \in 2^{\Theta}$. 125Here, $A_{i}$ designates a single hypothesis $H_{i}$ or union of simple hypotheses (composite hypotheses). 126An information source assigns mass values only to those hypotheses, for which it has direct 127 evidence. That is, if an information source can not distinguish between two propositions $A_{i}$ and $A_{j}$, it 128assigns a mass value to the set including both propositions $\left(A_{i} \cup A_{j}\right)$. This point is precisely the 129reason for us to choose the DS theory because it reflects the hesitation between two hypotheses.

130Obtaining the mass distribution or function $m\left(A_{i}\right)\left(0 \leq m\left(A_{i}\right) \leq 1 \quad\right)$ is the most important step since it 131represents the knowledge about the current application as well as the uncertainty and imprecision 132incorporated in the selected information source. The mass distribution for all the hypotheses has to 133fulfil the following conditions:

$134 m(\phi)=0$

$135 \sum_{A_{i} \in 2^{\ominus}} m\left(A_{i}\right)=1$

136Mass distributions $m_{1}, m_{2}$ from two different information sources are combined with Dempster's 137orthogonal rule. The result is a new distribution, $m=m_{1} \oplus m_{2}$, which carries the joint information 138provided by the two sources: 
140where

141

$K=\sum_{A p \cap A q=\varnothing} m_{1}\left(A_{p}\right) m_{2}\left(A_{q}\right)$

$142 \mathrm{~K}$ is often interpreted as a measure of conflict between the two sources and introduced in Equation 143(2) as a normalization factor. The larger the $K$, the more conflicting are the sources and the less 144sense makes their combination. As a consequence some authors, Smets in particular (Smets, 1990), 145require the use of the Dempster combination rule without normalisation. However, in this case, the 146relation (1) is no more verified. In our case, the normalised rule is used, and the K value is taken 147 into account in the decision process.

148In case of $\mathrm{N}$ different information sources $B_{1}, B_{2} \ldots B_{N}$, the DS rule is:

$149 m\left(A_{i}\right)=\frac{\sum_{B_{1} \cap B_{2} \cap \ldots \cap B_{N}=A_{i}} m_{l}\left(B_{1}\right) m_{2}\left(B_{2}\right) \ldots m_{N}\left(B_{N}\right)}{1-K}$

150where

$$
K=\sum_{B_{1} \cap B_{2} \cap \ldots \cap B_{N}=\phi} m_{l}\left(B_{1}\right) m_{2}\left(B_{2}\right) \ldots m_{N}\left(B_{N}\right)<1
$$

152From a mass distribution, two functions can be evaluated that characterize the uncertainty about the 153 hypothesis $A_{i}$. The belief function $\mathrm{Bel}$ measures the minimum uncertainty value about $A_{i}$ whereas 154plausibility $P l s$ reflects the maximum uncertainty value about this hypothesis. These two measures 155 span an uncertainty interval $\left[\operatorname{Bel}\left(A_{i}\right), \operatorname{Pl}\left(A_{i}\right)\right]$, which is called "belief interval". The length of this 156interval gives a measurement of imprecision about the uncertainty value. Belief and plausibility 157 functions are defined from the set of symbolic set of classes $2^{\Theta}$ to the unit interval $[0,1]$ :

$$
\operatorname{Bel}\left(A_{i}\right)=\sum_{A_{j} \subseteq A_{i}} m\left(A_{j}\right)
$$

$160 P l s\left(A_{i}\right)=\sum_{A_{j} \cap A_{i} \neq \phi} m\left(A_{j}\right)$

161These measures have been sometimes referred to as lower and upper probability functions.

162If only two hypotheses are used, the credibility of each single hypothesis equals its mass value, and 163the credibility of the combined hypotheses (which represents the ignorance value) equals 1 . This is 164the case for us when considering our frame of discernment: hypothesis $\mathrm{H}_{1}$ ("this object is a true 165defect TD"), hypothesis $\mathrm{H}_{2}$ ("this object is not a true defect", i.e. it is a false defect FD), and the 166ignorance is represented by the combined hypothesis $\left(\mathrm{H}_{1} \cup \mathrm{H}_{2}=\mathrm{H}_{3}\right)$. 
167Once data are combined, a decision rule must be applied. In our case, a threshold is applied on the 168 mass value corresponding to the $\mathrm{H}_{1}$ hypothesis. In the modelling step, specific care is taken in order 169 to avoid conflicts between sources.

\section{Proposed approach}

171

172The proposed new approach has the goal to automatically classify a detected object into either a TD 173or a FD, without expert supervision. This is done by analysing the features values extracted from 174each detected object, assigning to it a confidence level (or mass) and then combine mass values ob175 tained from different features.

\section{6}

177In the following, the term "object" refers to the result of the segmentation process, each object be178ing potentially either a true or a false defect. Both the ISAR system and our new classifier are based 179 on the same segmentation stage, so that the pre-processing does not influence the results (pre pro180cessing is explained in section 5.1).

181

182The proposed approach consists of a learning stage, and a classification stage, which are detailed 183 hereafter.

\section{4}

185The learning is done on a population of known and manually classified objects. For those objects, a 186set of features is computed, and the histogram of each feature is built. From these histograms, re187 gions of confidence are automatically defined with fuzzy transitions and masses are attributed, de188pending on the true defects proportion in the region. The complete procedure is detailed below.

190After this learning stage, for each object, a set of mass values is computed for each of its features. It 191 is then possible to consider each feature as a source of information about the set of hypotheses, and 192 combine them. Final decision is obtained by a threshold applied on the combined mass associated to 193the hypothesis "defect" $\left(\mathrm{H}_{1}\right)$.

\section{4}

\subsection{Learning}

\subsubsection{Estimation of true defect proportion}

199For a population of known detected objects (TD and FD), the set of values taken by a feature can be 200represented in the form of a spatial repartition. The histogram is used to divide this spatial reparti201tion into several regions of confidence. To illustrate the method, we use an individual normal dis- 
202tributed random variable $\mathrm{x}$ and two sets: set A representing the class of true defects TD, and set $\mathrm{B}$ 203representing the class of false defects FD (see figure 1). An instance of $\mathrm{x}$ can be classified into A or 204B. x can take any value of the codomain of a defect feature. This example will be used for illustra205 tion in each step.

206

207The histogram of feature distribution is plotted in figure 2 where a number of 100 intervals was 208 chosen in the feature values range in order to have a precise estimation.

209

210Let I be the set of intervals forming the feature's histogram. For each interval $i$

I, the percentage

211 of instances of the class A present in this region is calculated using the following function:

$212 P_{A, B}(i)=\frac{h_{A}(i)}{h_{A}(i)+h_{B}(i)}$

213

$214 h_{A}^{(i)}$ represents the number of instances of A inside $i$.

$215^{h_{B}(i)} \quad$ represents the number of instances of B inside $i$.

216

2173.1.2 Elaboration of regions of confidence and associated mass functions

218

219At first, each interval $i$ is considered as a region, and a set of mass functions is attributed to this in220terval:

221

$222 m\left(H_{1}\right)=P_{A, B}(i)$

$223 m\left(H_{2}\right)=0$

$224 m\left(H_{3}\right)=1-P_{A, B}(i)$

225

$226 m\left(H_{l}\right)$ is the mass assigned to the hypothesis: instance of A or TD.

227

228No mass is affected to the hypothesis $\mathrm{H}_{2}$ (instance of B) to ensure that no conflict occurs between 229 two sources of information in the fusion process.

$230 \mathrm{~m}\left(\mathrm{H}_{3}\right)$ is the mass assigned to the hypothesis ignorance (instance of A or B).

231

232Secondly, subsequent intervals are congregated to form a region of confidence. To be able to merge 233two intervals, the variation of the function $P_{A, B}$ is computed. Let $\sim_{A, B}(i)$ be the obtained function 234(see figure 3). 
236If the difference between the $P_{A, B}$ values $\quad \triangle_{A, B} \quad$ for two adjacent regions is less than a fixed threshold $237 \mathrm{~T}$ [first constraint], these two regions are merged and they will have in this case the same mass val238ues:

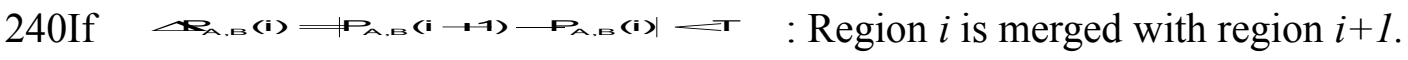

242Applying this method shows that the first constraint is not completely effective alone because some 243regions do not contain enough points to be considered as significant. For this reason a second con244 straint on the number of points existing in each region is imposed: a region should contain at least a 245 certain percentage of points [second constraint] to be considered as having enough significance. Ap246plying the merging procedure and ensuring enough points in each region, the obtained result is 247shown in Figure 4, where three regions of confidence are found. The $m\left(H_{l}\right)$ is specified for each re248gion.

\subsubsection{Definition of fuzzy transitions between regions}

252It can be observed in figure 4 that the mass value from one region to another may change greatly.

253 Therefore it is important to be able to represent the transition between two regions of the graph in a 254continuous manner. The theory of fuzzy sets is used to solve this issue, by building membership 255functions which allow the continuous passage from one region to another (Kaftandjian et al., 2003, 256Kaftandjian et al., 2005).

258Let $\mathrm{f}_{\mathrm{k}}$ be a source of information used to classify an object in class A or B according to its feature $x$, 259and $\mathrm{R} 1, \mathrm{R} 2, \ldots$, Rs be the set of regions of confidence. $\mathrm{k}=1 \ldots \mathrm{N}$ is the number of features considered 260as information sources.

261Each object has a set of degrees of membership $\mu_{\mathrm{i}}(\mathrm{i}=1 \ldots \mathrm{s})$ to each region $i$ with:

$$
\sum_{i=1}^{s} \mu_{i}(x)=1
$$

264Figure 5 illustrates the fuzzy transitions obtained for the regions of figure 4. A fuzzy set is defined 265and a membership function (classical trapezoidal shape) is built for each region. The slope of the 266 membership function is chosen to be proportional to the difference between the mass values of the 267adjacent regions. 


\subsection{Classification}

270

\subsubsection{Mass values attribution}

272

273The final mass attributed by a source $f_{k}$ to the object from its feature $x$ is calculated by weighting 274the mass values of each region $i$ by its degree of membership $\mu_{\mathrm{i}}$ :

$275 m\left(\right.$ object $\left.\in H_{1}\right)=m\left(x / f_{k}\right)=\sum_{i=1}^{s} \mu_{i}(x) \times m\left(R_{i}\right)$

276The final mass function obtained for the regions of figure 4 is represented in figure 5 . The mass as277signed to hypothesis $\mathrm{H}_{3}$ is the complement to 1 of the mass assigned to $\mathrm{H}_{1}$.

278

279

$m\left(\right.$ object $\left.\in H_{3}\right)=1-m\left(\right.$ object $\left.\in H_{1}\right)$

280

281Afterwards the fusion process of different sources takes place.

282

\subsubsection{Data fusion}

284

285The Dempster-Shafer rule is used to combine each two sources among N (see eq. 2), three most op286timistic sources for each object and the $\mathrm{N}$ sources (see eq. 3).

287

288Statistical fusion methods are also used by introducing: the mean mass value and the median mass 289 value.

290

291For an object, the mean mass is the mean value of all the mass values corresponding to this object. 292It gives a sort of equal influence to the different opinions.

293

294The median mass is the median value of all the mass values corresponding to an object. It allows to 295 introduce a majority decision for the cases where one or few sources are not in accordance with the 296others.

297

\subsubsection{Decision}

299

300To classify an object using the information source $f_{k}$, a threshold $S$ is applied on its mass value $301 m\left(H_{l}\right)$ (which represents here the credibility of the hypothesis as said in section 2).

302The object is classified as: 
303- a defect if $m\left(H_{l}\right) \geq S$.

304- unknown (defect or not) if $m\left(H_{l}\right)<S$.

305

3064. Performance measurements and choice of optimal sources

307

308Classification rates are introduced to measure the performance of the different combinations of 309information sources. These measures are calculated for different thresholds $S$ applied on the mass 310 values. For a certain threshold $S$, an object with mass $m\left(H_{l}\right)$ is correctly classified if the source 311decision is in accordance with the human decision, this means:

312- the object is a true defect (human decision) and its corresponding mass value is higher or equal to 313the threshold $m\left(H_{l}\right) \geq S$.

314- or it is a false defect (human decision) and its mass is lower than the threshold $m\left(H_{l}\right)<S$.

315

316We introduce four measures:

317

318- Correct decisions rate $(\mathrm{PCD})$ :

$$
\text { PCD }=\frac{\text { number of true defects correctly classified }+ \text { false defects correctly classified }}{\text { total number of true defects and false defects }}
$$

321- True Defects detection rate (PTD):

$$
\text { PTD }=\frac{\text { number of true defects correctly classified }}{\text { total number of true defects }}
$$

324- False Defects detection rate (PFD):

330- Overall detection rate $\mathrm{R}$ : 
333The rate $\mathrm{R}$ was introduced in such a way to give the user the possibility to attribute more import334 ance to the correct classification of either TD or FD. Usually more importance is given to TD detec335tion.

337Using these measures allows comparing the final performance of the method with the existing in338spection system ISAR introduced in the next section.

\section{Experimental results}

342This section is devoted to present the practical application of our new method. The pre-processing 343 stage and features are described first, then the data base is presented and analysis of the results after 344 data fusion is finally detailed.

\section{5.1 Pre-processing stage and selected features}

348ISAR is a fully automatic system for radioscopic quality control currently in use in the production 349 of castings. Within the database used in the present paper, evaluation is done by using a reference 350image, because thus a higher sensitivity to small defects is possible (Fuchs et al, 2006). The data 351 processing can be separated into five stages: registration, calibration, image processing, fault 352 segmentation, and quality assessment.

$353 \cdot$ Registration

354Each inspected part that is processed during inline testing is not necessarily measured at exactly the 355 same position. Due to mechanical instabilities, long-term changes of the moulds and slight 356differences between the moulds in use, an automatic registration step is needed, where the current 357 test image is registrated to the appropriate reference image using an affine transformation.

$358 \quad$ Calibration

359In order to allow for quantitative measurement of the depth of faults, i.e. to extract their length in 360direction of the penetrating radiation, a physical calibration object is evaluated. The necessary 361 characteristic line is created using a step-wedge made out of the same material as the part and with 362 typically 10 to 15 steps covering the maximum thickness of the objects to be tested.

363 - Image processing

364The reference image is subtracted from the current image. Thereby, all structures that do not appear 365 within the fault-free reference part will become clearly visible, that is to say potential defects. In 366case of improper registration results however, edges due to the object structure may remain in the 367 subtraction image, which is one cause of false defects.

$368 \quad$ Segmentation

369The segmentation step is to assemble the single suspicious pixels into well defined fault regions 370 appropriately. The analysis is based on the subtraction image. As result of this operation, we will 371 have for each detected region several features that describe this object and which will be used for 372 defect classification. Each detected object in this stage is classified by ISAR as true defect or false 373 defect, and our new classification system starts with the same features.

374 Quality assessment

375Quality criteria are applied by ISAR to the identified defects, which are defined by the end user for 376a final decision quality of the part (e.g. acceptance based on size or shape of defects). This post377 processing was not included in our system. 
379The features which are selected are described as following:

380 - Area $\left[\mathrm{mm}^{2}\right]$ : is defined as the number of pixels of the detected object multiplied by the size of

381 the pixel defined by the acquisition geometry.

382 - Depth [mm]: the main objective of using the length image is to precise the size of the defect in

383 X-ray penetration direction. Depth is defined as the mean value of the pixels of the detected

384 object.

385 -Volume $\left[\mathrm{mm}^{3}\right]$ : is calculated using the area occupied by this object multiplied by its depth.

386 - MaxDepth [mm]: is the highest value inside the defect region.

387 - DepthDev: is equal to standard deviation of the defect's depth in the defect region.

- InOutContrast [mm]: is also measured on the length image and is equal to InOutContrast $=\mid$ mean (defect region) - mean(surrounding region) $\mid$

- MaxElongation [mm]: is defined as the diameter of the minimum covering circle of the detected object.

- Diff1stDev: This feature represents the difference, inside the defect region, of the mean gradient value within the reference image to the mean gradient value within the current image.

- InOutContrastGV: is the same as InOutContrast but in grey values instead of $\mathrm{mm}$.

- Thickness [mm]: is the thickness of the part inside the defect region.

- Depth2Thickness: is the ratio between the depth of the object and the thickness of the part in 399 the corresponding region.

\subsection{Data base description}

401

402In this work a database is extracted from industrial images. The detected objects, also called poten403tial defects, are classified manually into TD and FD.

404

405The database is formed of 597 objects. It contains 382 true defects including oxides, gas voids and 406porosities and 215 false defects.

407

408The database was divided into two parts:

409-Learning database: formed of 115 false defects and of 243 true defects.

410-Testing database: formed of 100 false defects and 139 true defects.

\subsection{Application: Learning and classification}

416Spatial repartitions of four features (Area, Depth, InOutcontrast and Thickness) corresponding to 417the objects of the learning database are presented in figure 6. All the other features present their own 418spatial repartition with some overlapping between TD and FD classes. The Area graph exhibits the 
419better discrimination between classes with true defects mainly having small areas, although some 420 false defects also.

423First the estimations of the features histograms take place. Figure 7 illustrates the histograms of the 424four features represented in figure 6. As already mentioned, overlapping between TD and FD 425 classes is important.

426

427Using the feature's histogram the regions of confidence are built. The results for the four previous 428features are shown in figure 8 where different number of confidence regions are identified. The val429ues on the right correspond to the mass values $m\left(H_{l}\right)$ attributed for each point falling into the corres430ponding region. In addition, membership functions were built for each feature (see figure 9).

432Using the estimated regions of confidence, their corresponding mass and membership functions, the 433single mass values of the learning database objects are computed (eq. 6). Mass functions obtained 434 for the four previous features are also shown in figure 9.

435

436Afterwards the fusion process takes place. As an example, the histogram of mass values obtained 437 from the combination of Area and InOutContrast is presented in figure 10. It can be observed that 438the translation from feature values to mass values results into an easier separation between TD and 439FD. Moreover, the fact that the mass values are included between 0 and 1 facilitates the interpreta440tion.

441Subsequently, the classification of the learning database objects using different thresholds on the 442 mass values given by each source (single and combined sources) is performed.

\subsection{Performance results: Learning database}

446The performance measures for ISAR on the learning database are: $\mathrm{PTD}=0.978, \mathrm{PFD}=0.723$ and $447 \mathrm{R}=0.928$ (with $\mathrm{a}=\mathrm{c}=1, \mathrm{~b}=5$ ). This choice of parameters $\mathrm{a}, \mathrm{b}$ and $\mathrm{c}$ allows to give more importance to 448the classification of true defects, which is an industrial requirement.

449Individual features performance after masses attribution is shown in Table 1. As it can be noticed, 450some of the features are better than the others (Area in particular, as was visible from its histogram), 451 nevertheless, all of them will be kept for fusion due to the fact that they could bring some informa452tion. 
454ISAR has, in the tested configuration an excellent performance in the detection of true defects but it 455 has a false detection rate of about $28 \%$. After the data fusion process, PTD, PFD and R are meas456ured for all the single and combined sources (total of 70 sources). Several combined sources give 457 better classification rates compared to the original ISAR decision (see figure 11). All these combin458ations present better PTD, PFD and R than ISAR. Grouped into a set $\varepsilon_{c}$, they are then used as 459 sources of information to classify the objects of the testing database.

460

461The best classification performance as measured by the overall rate $\mathrm{R}$ is obtained by the DS com462bination of the features Area and Depth with $96 \%$, for a threshold on the mass values $S=0.8$, instead 463of 92\% for ISAR. Table 2 gives the results obtained for PTD and PFD of this combination which 464are all higher than ISAR's measures.

465

466When considering the performance of true defects classification only, the best source is the DS 467combination of Area and Thickness with PTD of $99 \%$, for the threshold S=0.9, instead of $97 \%$ for 468ISAR. Here also PFD and R are both higher than ISAR's measures (see table 3).

469

470The best performance for false defects classification only is obtained by the mean mass with a PFD 471 of $98 \%$, for the threshold $\mathrm{S}=0.7$, which is $28 \%$ better than ISAR's PFD. However, PTD is in this 472case lower than ISAR's PTD which is not acceptable for this application (see table 4), therefore the 473 mean mass is not presented in figure 11. Nevertheless, this source will be considered for testing.

474

\subsection{Validation: Testing database}

476

477The testing database is formed of 139 true defects and 100 false defects.

478

479ISAR shows similar performance on the testing database with: $\mathrm{PTD}=0.980, \mathrm{PFD}=0.725$ and $480 \mathrm{R}=0.930$.

481

482After the learning step, the set of sources $\Sigma_{L}$ are used to automatically attribute mass values for 483the new objects of the testing database. Afterwards data fusion and classification take place.

485All combined sources improve with an important percentage the classification of false defects and 486also are equal or even exceed ISAR performance on true defects detection (see figure 11 (b)). In the 487learning database, the optimal overall source was the fusion between Area and Depth. On the testing 488database, this combination presents a rate $\mathrm{R}$ of $96.37 \%$ (for the threshold $\mathrm{S}=0.8$ ), instead of $93.07 \%$ 
489for ISAR, with a very small difference from the optimal overall source on the testing database 490which is the fusion between Area and MaxElongation with $\mathrm{R}=96.86 \%$ (see table 5).

491

492 The optimal combination for the detection of true defects in the learning database (DS combination 493of Area and Thickness) is still the best combination for the detection of true defects in the testing 494database with a PTD of $0.99 \%$ for the threshold $S=0.9$ (see table 6). The improvement in the detec495tion of true defects is $1 \%$ relatively to ISAR, and $12 \%$ in the detection of false defects. The optim496al combination for the detection of false defects in case of testing database is still the mean mass 497value for the threshold $S=0.7$ (see table 7). The improvement in the detection of false defects is 25.8 $498 \%$ relatively to ISAR, but a loss of almost $4.5 \%$ in the percentage of true defects detection occurs. 499To illustrate two cases where ISAR fails, while data fusion gives the good result, two objects are 500 chosen. Figure 12a shows a false defect classified as a true one, and figure $12 \mathrm{~b}$ shows a true defect 501classified as a false (the object appears brighter than the background inside the rectangular zone).

502

503

504

\section{Discussion}

506

507The first point to emphasize is that the results obtained during the testing phase confirm the 508robustness of the method. All sources selected during learning keep their high performance during 509testing.

511In our previous work (Kaftandjian et al., 2003) only 4 features were selected as best candidates to 512 discriminate between TD and FD. This selection of features (contrast to noise ratio, area, elongation 513 and position) was done by the expert based on his knowledge of X-ray inspection. The drawback 514 was to require the expert's supervision during learning. Here the approach is completely automated 515 and all the features are considered without a priori information. The obtained performance cannot 516be compared with (Kaftandjian et al., 2003) due to the fact that the application was not the same.

517When considering which features give the best classification rates, one can be surprised that the 518 contrast feature is not among the best ones, although contrast is usually considered as having the 519main physical meaning. Indeed it is impossible to justify a posteriori why some features were better 520than the others when combined. This is due to the fact that our knowledge is only valid for TD, and 521the classification of FD cannot be based on a physical basis. This is precisely the difficulty of FD 522detection and the reason why even the expert find them difficult to classify. Therefore an important 523advantage of our method is that it adapts itself to all the objective data that are measured on the true 
524and false defects, without any a priori knowledge.

525

526Another fact to mention is that the developed method is general in the sense that it can work for any 527inspection method. The translation from the feature space to the mass values space allows to 528 combine any information sources independently of their origin (ultrasonic data, radiographic 529 data...).

530

\section{7. Conclusion}

532

533A new implemented method able to automatically detect regions of confidence from a feature's 534histogram and to affect degrees of confidence (mass values) to these regions was presented. The 535automatic learning process is based on a classified database. The method consists in translating a 536feature value into a confidence or mass value. These mass values are used within the fusion process. 537The method was applied on a learning database and validated on a testing database for a casting 538application. As could be shown, several feature combinations are exceeding the current ISAR 539decision. Using the data fusion theory, while conserving at least the $98 \%$ percentage of 540classification of TD given by ISAR, an improvement from $72 \%$ to $92 \%$ (see table 4 ) in the 541 percentage of FD classification was achieved. An improvement of $3.8 \%$ of the overall rate $\mathrm{R}$ is 542obtained.

544Some combinations can optimize even better either the TD or the FD classification. A PTD rate of $54599 \%$ and PFD of $98.3 \%$ was obtained separately. Further work is needed to find an optimal decision 546rule which would associate the best TD and FD classification rates.

548In a future work, a study of the robustness of this method for another application will be done. 549Further investigation will also be conducted to measure the influence of the constraints on the 550derivative variation and on the percentage of points inside the regions of confidence on the results.

\section{Acknowledgements}

553

554This work was co-financed by the European Union and the Free State of Bavaria within the project 555ZeLuR. 
560Bonser G., Lawson S.W., "Defect detection in partially complete SAW and TIG welds using on561line radioscopy and image processing”. Proceedings of SPIE International Symposium on Non 562Destructive Evaluation Techniques for Aging Infrastructure and Manufacturing, pp. 231-239, 1998, 563San Antonio Texas USA.

564

565Dempster A., "Upper and lower probabilities induced by multivalued mapping", Annals of 566Mathematical Statistics, 38, pp. 325-339, 1967.

567

568Dobrazanski L.A., Krupinski M., and Sokolowski J.H., "Computer aided classification of flaws 569occurred during casting of aluminum". Journal of Materials Processing Technology, Vol. 167, 570Issues 2-3, pp. 456-462, August 2005.

571

572Fuchs T., Hassler U., Huetten U., and Wenzel T., "A new system for fully automatic inspection of 573digital flat-panel detector radiographs of aluminium castings". Proceedings of 9th European 574Conference on Non-Destructive Testing (ECNDT), Sept. 25 - 29, 2006, Berlin Germany.

575

576Hernandez S., Saez D., Mery D., Silva R., and Sequeira M., "Automated defect detection in 577aluminum castings and welds using neuro-fuzzy classifiers".16th World Conference on NDT, Aug 57830 - Sep 3, 2004, Montreal Canada.

579

580Jang J., "Anfis: Adaptive-network-based fuzzy inference system" IEEE Transactions on Systems, 581Man, and Cybernetics, Vol. 23, No. 3, pp. 665-684, 1993.

582

583Kaftandjian V., Dupuis O., Babot, D. and Zhu Y., "Uncertainty modeling using Dempster-Shafer 584theory for improving detection of weld defects". Pattern Recognition Letters, Volume 24, pp. 547585564, 2003.

586Kaftandjian V., Zhu Y.M., Dupuis O., Babot D., The combined use of the Evidence Theory and Fuzzy 587Logic for improving multimodal Non Destructive Testing systems, IEEE Trans. On Instrumentation \& 588Measurement, 2005, vol.54, n5, p.1968-1977.

590Lawson S.W. and Parker G.A., "Intelligent segmentation of industrial radiographic images using 591neural networks". Machine Vision Applications and Systems Integration III, Proceedings of SPIE, 592pp. 245-255, November 1994. 
594Lecomte G., Kaftandjian V., and Cendre E., "Combination of information from several x-ray 595images for improving defect detection performances-application to castings inspection". 596Proceedings, 9th European Conference on Non-Destructive Testing (ECNDT), Sept. 25 - 29, 2006, 597Berlin Germany.

598

599Mery D. and Filbert D., "Classification of potential defects in the automatic inspection of aluminum 600castings using statistical pattern recognition". In Proceedings of the 8th European Conference on 601Non-Destructive Testing (ECNDT 2002), June 2002, Barcelona Spain.

602

603Newman T. and Jain A., "Bidirectional template-matching for 3d cad-based inspection". 604Proceedings of SPIE Machine Vision Applications in Industrial Inspection II, San Jose, pp. 257605265, February 1994.

606

607Shafer G., "A mathematical theory of evidence”, Princeton University Press, Princeton, pp. 297, 6081976.

609Smets P., "The combination of evidence in the Transferable Belief Model”, IEEE trans. Pattern 610Analysis\& Machine Intelligence, vol 12, n5, pp. 447-458, 1990.

611

612

613Theis G. and Kahrs T., "Fully automatic X-ray inspection of aluminum wheels". Proceedings of the 6148th European Conference on Non-Destructive Testing (ECNDT 2002), June 2002, Barcelona Spain. 615

616Wenzel T. and Hanke R., "Texture analysis for classification of cast defects in x-ray images". $2^{\text {nd }}$ 617workshop "NDT in progress" International Meeting of NDT Experts, October 6-8, 2003, Prague, 618Czech Republic.

619

620

621

622

623

624

625

626

627

\section{$628 \underline{\text { Captions }}$}


630Fig. 1:

631Two normal distributions where a star point represents a member of the class A and a cross point 632represents a member of class B.

\section{Fig. 2:}

634Feature's histogram of class A (full line) and class B (dotted line).

635Fig. 3:

636Illustration of $\quad P_{A, B}(i) \quad$ (full line) and $\quad \sim_{A, B}(i) \quad$ (dotted line) corresponding to the histogram of fig637ure 2.

638Fig. 4:

639Regions of confidence after merging and their corresponding mass values $m\left(H_{l}\right)$.

640Fig. 5:

641Membership functions corresponding to the regions of figure 4 (full line) and mass functions (dot642ted line).

\section{Fig. 6:}

644Spatial repartition of True and False defects for Area (high left), Depth (high right), InOutContrast 645(low left) and Thickness (low right)

\section{Fig. 7:}

647Histograms for Area (high left), Depth (high right), InOutContrast (low left) and Thickness (low 648right).

\section{Fig. 8:}

650Regions of confidence and corresponding mass values $\mathrm{m}\left(\mathrm{H}_{1}\right)$ for Area (high left), Depth (high 651right), InOutContrast (low left) and Thickness (low right).

\section{Fig. 9:}

653Membership functions (solid line) and mass functions (dotted line) corresponding to the confidence 654regions of Area (high left), Depth (high right), InOutContrast (low left) and Thickness (low right)

\section{Fig. 10:}

656 Histogram of mass values obtained from the combination of Area and InOutContrast. The majority 657 of true defects have mass values close to one.

\section{Fig. 11:}

659Classification rates obtained on the learning database (a) and on the testing database (b). The selec660ted sources are those giving a better overall classification rate than ISAR, and the same set of 661 sources are still better on the testing database

662

663Fig. 12: 
664False defect classified as a true one by ISAR (a) and true defect classified as false (b). In those two 665 cases, data fusion give the good result. The true or false defects appear as a brighter zone in the 666rectangle

667

668Table 1:

669Performances of single features after mass attribution, in the learning database. Features are classi670 fied with respect to the overall rate $\mathrm{R}$

671

672Table 2:

673Combination giving the highest overall rate $\mathrm{R}$ in the learning database.

674

675Table 3:

676Combination giving the highest true defects detection rate PTD in the learning database.

677

678Table 4:

679Combination giving the highest false defects detection rate PFD in the learning database. 680

681Table 5:

682 Combination giving the highest overall rate $\mathrm{R}$ in the testing database.

683

684

685Table 6:

686Combination giving the highest true defects detection rate PTD in the testing database.

687

688Table 7:

689Combination giving the highest false defects detection rate PFD in the testing database.

690

691 